\title{
Futuros professores de matemática e o ensino de proporcionalidade através da resolução de problemas de geometria
}

\author{
Manoel dos Santos Costa \\ Professor, Instituto de Ensino Superior Franciscano e da SEE/MA \\ manolopromat@hotmail.com
}

\author{
Norma Suely Gomes Allevato \\ Professora, Universidade Cruzeiro do Sul / SP \\ normallev@uol.com.br
}

\begin{abstract}
Resumo
O presente estudo tem como objetivo descrever e analisar como (futuros) professores de Matemática aplicam o conceito de proporcionalidade para calcular termos desconhecidos de um problema de Geometria que envolve o Teorema de Tales. O estudo é de natureza qualitativa e os dados foram coletados através de observações durante encontros de formação em que os (futuros) professores vivenciaram a resolução de problemas como metodologia de ensino. Os resultados mostram que no decorrer das discussões os (futuros) professores mudaram de opinião com relação à metodologia de ensino-aprendizagem-avaliação através de resolução de problemas. Além disso, a pesquisa também revelou que os (futuros) professores apresentaram deficiências e construíram conhecimento com relação ao conteúdo estudado, principalmente, às conexões com outras áreas da Matemática - neste estudo, com a Geometria. E não apenas com relação aos conteúdos; eles também puderam discutir aspectos relacionados a "quando" e "como" deveriam ensinar proporcionalidade.
\end{abstract}

Palavras chave: Proporcionalidade. Geometria. Futuros Professores. Resolução de Problemas. Educação Matemática.

\section{Future teachers of mathematics of proportionality through problem solving in geometry}

\begin{abstract}
The present study aims to describe and analyze how (future) Mathematics teachers in training apply the concept of proportionality to calculate unknown terms of a Geometry problem involving the Theorem of Thales. The study was qualitative and data were collected through observation during training meetings in which the (future) teachers experienced problem solving as a teaching methodology. The results show that along the discussions the (future) teachers changed their view regarding the methodology of teaching-learning-evaluation through problem solving. In addition, the research also revealed that the (future) teachers had disabilities and built knowledge regarding the content studied, mainly the connections with other areas of Mathematics in this study, with Geometry. And not just with respect to contents, but they could also discuss issues related to "when" and "how" they should teach proportionality.
\end{abstract}

Keywords: Proportionality. Geometry. Future teachers. Problem Solving. Mathematics Education. 


\section{Introdução}

Este artigo é parte de uma pesquisa maior que se encontra em andamento e que foi realizada durante alguns encontros oferecidos a (futuros) professores que buscavam recursos para uma nova forma de desenvolver os conteúdos de Matemática em suas salas de aula. Nos encontros foi empregada a Metodologia de Ensino-Aprendizagem-Avaliação de Matemática através da Resolução de Problemas.

Particularmente, para o desenvolvimento deste trabalho analisamos as atividades de resolução de um problema de Geometria envolvendo o conceito de proporcionalidade, cujo objetivo era verificar como (futuros) ${ }^{1}$ professores em formação inicial aplicam o conceito de proporcionalidade (propriedade fundamental das proporções) para calcular termos desconhecidos de um problema de Geometria que envolve o Teorema de Tales

O presente trabalho está organizado em seções, iniciando pelas abordagens de resolução de problemas, destacando-a como uma metodologia de ensino de Matemática em que o problema é ponto de partida para a construção de conhecimento. A segunda seção aborda a proporcionalidade e seu papel de integrador entre os conteúdos matemáticos. Na terceira seção tratamos do contexto e da metodologia da pesquisa, assim como dos instrumentos utilizados. Na quarta seção, intitulada "Aprendendo o conceito de proporcionalidade através de resolução de problemas de Geometria", descrevemos e analisamos as atividades que (futuros) professores desenvolveram em um dos encontros de formação. Finalmente, apresentamos nossas reflexões finais.

\section{Resolução de problemas como metodologia de ensino-aprendizagem- avaliação}

O Ensino-Aprendizagem-Avaliação de Matemática através da Resolução de Problemas, tal como é apresentada por Onuchic e Allevato (2009), é uma metodologia diferente daquele trabalho em que regras de "como fazer" são privilegiadas. Trata-se de uma metodologia onde o problema é ponto de partida e orientação para a aprendizagem, e a construção do conhecimento far-se-á através de sua resolução.

Discussões no campo da Educação Matemática buscam melhores formas de ensinar e aprender Matemática. Há mais de três décadas, nos Estados Unidos, o $\mathrm{NCTM}^{2}$ já recomendava que "resolver problemas deve ser o foco da Matemática

1. Os parênteses são utilizados porque, embora os professores participantes da pesquisa fossem oficialmente alunos de um curso de licenciatura e, portanto, se encontrassem em formação inicial, alguns já atuavam efetivamente como professores em sala de aula.

2. NCTM - National Council of Teachers of Mathematics (Conselho Nacional de Professores de Matemática) - organização profissional, para professores de Matemática nos EUA sem fins lucrativos. 
Escolar para os anos 80" (NCTM, 1980, p.1). A partir daí, muitos estudos e elaboradores de orientações curriculares têm recomendado a resolução de problemas como metodologia de ensino.

No Brasil, documentos oficiais (MARANHÃO, 2007; BRASIL, 1998), e pesquisadores (ONUCHIC; ALLEVATO, 2009) apresentam algumas ações que podem ser praticadas no ensino de Matemática, destacando que a resolução de problemas "constitui uma metodologia voltada para a utilização de conceitos matemáticos pelo educando, por meio de situações-problema que estimulem a curiosidade, a investigação, a descoberta, a elaboração e reelaboração de conceitos que foram usados na solução do problema”. (MARANHÃO, 2007, p. 123).

Os PCN - Parâmetros Curriculares Nacionais (BRASIL, 1998) apontam o desenvolvimento da capacidade de resolver problemas, explorá-los, generalizá-los e até propor novos problemas a partir deles, como propósitos do ensino de Matemática; indicam a resolução de problemas como ponto de partida das atividades matemáticas e discutem caminhos para se fazer Matemática na sala de aula.

Alguns pesquisadores (ONUCHIC, 1999; ALLEVATO; ONUCHIC, 2009; NUNES, 2010; COSTA; ALLEVATO, 2010) utilizam a resolução de problemas nessa linha, constatando que importantes conceitos e procedimento podem ser melhor ensinados se utilizada a Metodologia de Ensino-Aprendizagem-Avaliação de Matemática através da Resolução de Problemas. Essa metodologia designa uma abordagem onde a construção de conhecimento se faz a partir de "problemas geradores", propostos como ponto de partida e orientação para a aprendizagem de novos conceitos e novos conteúdos.

Mas o que significa ensinar, aprender e avaliar Matemática através de resolução de problemas? A palavra composta ensino-aprendizagem-avaliação expressa uma concepção em que o ensino, a aprendizagem e a avaliação devem ocorrer simultaneamente durante a construção do conhecimento de um determinado conteúdo através da resolução de problemas (ALLEVATO; ONUCHIC, 2009).

E o que vem ser um problema?

Onuchic (1999) esclarece sua compreensão, dizendo que um problema "[...] é tudo aquilo que não se sabe fazer, mas que se está interessado em resolver". A autora ainda esclarece que "o problema não é um exercício no qual o aluno aplica de forma quase mecânica uma fórmula ou uma determinada técnica operatória". (ONUCHIC, 1999, p. 215), mas exige a elaboração de estratégias que possibilitem o aprimoramento do conhecimento durante a construção de sua resolução.

Segundo Vianna (2002), um problema é individual, para cada pessoa, condicionado àquilo que é o seu mundo e às suas preocupações. $\mathrm{O}$ autor afirma: um problema é "uma situação em que um sujeito é solicitado a realizar uma tarefa para 
a qual não possui um método de resolução determinado. Se a realização da tarefa não for desejada pelo sujeito a situação não pode ser considerada um problema" (VIANNA, 2002, p. 403).

Sendo assim, como as colocações de Vianna (2002) reforçam e complementam a definição de Onuchic (1999) para um problema, assumimos essas duas definições para o nosso trabalho.

Portanto, ensinar Matemática utilizando resolução de problemas não é uma tarefa fácil, pois não basta apresentar um problema e "ficar sentado" esperando que alguma mágica aconteça. $\mathrm{O}$ professor precisa criar e manter um ambiente motivador e estimulante. Por isso, as aulas devem acontecer passando por três momentos: o antes, o durante e o depois. No primeiro momento o professor deve garantir que os alunos estejam prontos para receber o problema e que as expectativas estejam claras; isto significa que, neste momento o professor identifica aquilo que o aluno entendeu e a partir daí ativa seus conhecimentos prévios. No segundo momento, "o durante", o professor deverá observar e estimular o trabalho de resolução dos problemas pelos alunos, ou seja, o professor deixa que o aluno construa seus conhecimentos, evitando antecipações desnecessárias. E no último momento o professor deverá analisar as soluções apresentadas pelos alunos sem julgá-las como certas ou erradas, mas levando-as a uma discussão (plenária) para que o aluno possa justificar e avaliar seus próprios métodos e com isso, validar os resultados obtidos. No final, o professor formalizará os novos conceitos e conteúdos construídos a partir e durante a resolução do problema (VAN DE WALLE, 2009).

Considerar a resolução de problemas como metodologia de ensino, não significa dizer que existe uma forma rígida para desenvolvê-la nas aulas de Matemática. No entanto, Allevato e Onuchic (2009) sugerem algumas etapas para que se possa colocar em prática e usufruir melhor dessa metodologia:

(1) Preparação do problema - Selecionar um problema visando à construção de um novo conceito, princípio ou procedimento. (2) Leitura individual - Entregar o problema para cada aluno e solicitar que seja feita sua leitura. (3) Leitura em conjunto - Solicitar nova leitura do problema, agora em pequenos grupos de alunos. (4) Resolução do problema - De posse do problema, sem dúvidas quanto ao enunciado, os alunos, em seus grupos, buscam resolvê-lo. (5) Observar e incentivar - $\mathrm{O}$ professor não é mais transmissor do conhecimento. Enquanto os alunos, em grupo, buscam resolver o problema, o professor observa, analisa o comportamento dos alunos e estimula o trabalho colaborativo. Como mediador, leva os alunos a pensar, dando-lhes tempo e incentivando a troca de idéias entre eles. (6) Registro das resoluções na lousa - Representantes dos 
grupos registram, na lousa, suas resoluções. (7) Plenária - Todos os alunos são convidados a discutir as diferentes resoluções registradas na lousa, defender seus pontos de vista e esclarecer suas dúvidas. (8) Busca do consenso - Sanadas as dúvidas e analisadas as resoluções e soluções obtidas para o problema, o professor tenta, com toda a classe, chegar a um consenso sobre o(s) resultado(s) correto(s). (9) Formalização do conteúdo - Neste momento o professor registra na lousa uma apresentação formal do conteúdo matemático, organizada e estruturada em linguagem matemática, padronizando conceitos, princípios e procedimentos construídos através da resolução do problema.

Essas etapas, ou adaptações delas, ajudam o professor a desenvolver a metodologia de ensino-aprendizagem-avaliação através de resolução de problemas; a identificar aquilo que os alunos sabem ou não sabem e, com isso, definir os objetivos de futuros problemas. Além disso, nesta metodologia de ensino a preocupação do professor não é com a apresentação da solução correta de imediato, mas com o processo de resolução. É importante que os alunos sejam estimulados a resolver o problema apresentado, recorrendo a e refletindo sobre seus conhecimentos prévios. O ideal é que o consenso sobre a solução correta seja atingido em discussão com toda a classe, na plenária. E ao final, o professor apresenta a formalização do conteúdo estudado partindo das resoluções apresentadas pelos alunos e podendo definir outros instrumentos e recursos a serem utilizados: textos, livros didáticos, anotações, entre outros, aos quais os alunos poderão recorrer para fixar, esclarecer e ampliar o conteúdo explorado.

Reiteramos que, nessa metodologia, os problemas são propostos aos alunos antes de lhes ter sido apresentado formalmente o conteúdo matemático mais apropriado à resolução do problema. Porém esse conteúdo deve estar de acordo com o ano escolar em que se encontram os alunos e com os objetivos pretendidos pelo professor para aquela aula. Assim, o ensino-aprendizagem do tópico matemático começa com o problema, que expressa aspectos-chave desse tópico, e técnicas matemáticas serão desenvolvidas na busca de respostas ao problema dado; a avaliação é feita continuamente, durante a resolução do problema.

E mais, essa metodologia contempla ações pedagógicas (interação entre aluno e professor) que promovem a busca por informação, a investigação, a experimentação e a renovação do interesse e da motivação dos alunos.

\section{A proporcionalidade e seu papel integrador}

A escolha pelo tema proporcionalidade deve-se à importância que é atribuída a este conceito, que é um conceito unificador na Matemática. "Ele aparece sob 
os mais distintos aspectos, ora em problemas aritméticos, como os de regra de três, ora em Geometria, como no Teorema de Tales, sem que o aluno perceba a menor relação entre eles" (TINOCO, 1996, p. 9). Nesta pesquisa, optamos por abordá-lo relacionado à Geometria, por envolver assuntos relevantes não somente aos alunos, mas também para os professores que, muitas vezes, não sabem como trabalhar com eles.

Segundo Costa e Allevato (2009) em virtude de a proporcionalidade ter esse papel integrador entre os diversos conteúdos matemáticos, trabalhar através de resolução de problemas pode ser um facilitador para os alunos conhecerem e compreenderem melhor o ensino de Geometria. Essa metodologia de ensino vem se destacando como sendo muito eficaz para desenvolver o raciocínio, para motivar os alunos e para proporcionar o estudo de novos conceitos e conteúdos da Matemática.

Normalmente, nas escolas, o conceito de proporcionalidade é apresentado aos alunos que estão cursando o $7^{\circ}$ ano do Ensino Fundamental, sendo priorizada a regra de três como meio para a resolução. Segundo Oliveira e Santos (1999)

No Brasil, o estudo da proporcionalidade ocorre, muitas vezes, de uma maneira fragmentada, onde cada assunto do capítulo referente ao tema proporcionalidade é visto como objeto de estudo em si mesmo, provocando a transformação de ferramenta de resolução a objeto de estudos, o que ocorre, especificamente, com a regra de três (OLIVEIRA; SANTOS, 1999, p. 2).

O conceito de proporcionalidade está presente no dia a dia de qualquer pessoa, nas mais diversas situações, envolvendo desde interpretar estatísticas e gráficos até fazer análises de plantas de imóveis ou mapas, ampliar ou reduzir fotos, entre outras. Ele também é essencial ao aprendizado de diversas disciplinas dos ensinos Fundamental, Médio e Superior: "São inúmeras as aplicações desse conceito à geografia, à física, à química, etc.” (TINOCO, 1996, p. 9).

Neste trabalho provocamos nos (futuros) professores uma instabilidade de forma que analisamos como eles percebem a relação existente entre a proporcionalidade e a Geometria através da resolução de problemas, dando um tratamento adequado e integrado a esses dois assuntos, por meio da reconstrução do conceito de proporcionalidade que, segundo Tinoco (1996), nos permite:

- construir uma visão mais unificada da matemática, onde a geometria, a álgebra e a aritmética não sejam tratadas como matemática distinta, como geralmente acontece; 
- valorizar a geometria, antecipando tópicos como o Teorema de Tales, para melhor relacioná-los com os outros ramos da matemática. (TINOCO, 1996, p. 9)

\section{Contexto e metodologia da pesquisa}

Os sujeitos desta pesquisa foram estudantes do curso de licenciatura em Matemática de um programa de formação inicial de professores de uma universidade pública no Estado do Maranhão. Esses estudantes reuniam-se semanalmente com o pesquisador para discutir e analisar como (futuros) professores de Matemática, em formação inicial, percebem a possibilidade de explorar o conceito de proporcionalidade através de resolução de problemas de Geometria

Durante a coleta dos dados realizamos leituras e discussões de textos sobre resolução de problemas e sobre o ensino de proporcionalidade e de Geometria, além de atividades práticas de resolução de problemas envolvendo esses conteúdos. As resoluções dos problemas, as atividades escritas produzidas pelos participantes e as discussões, registradas em um diário de campo, constituem os dados desta pesquisa.

A pesquisa é de natureza qualitativa. Grande número de pesquisadores da área de Educação tem demonstrado interesse por esse tipo pesquisa, pois coloca-os em contato direto com o ambiente da pesquisa, com os sujeitos envolvidos e com os problemas que estão sendo estudados. Esse contato ocorre, normalmente, quando se realiza a pesquisa de campo. (LUDKE; ANDRÉ, 1986). Na pesquisa aqui relatada, o pesquisador foi o responsável pela organização e condução das atividades desenvolvidas. Em todos os momentos, a atenção foi colocada nos processos utilizados pelos participantes no desenvolvimento das atividades de resolução dos problemas e não somente nos resultados (GOLDENBERG, 2007).

\section{Aprendendo o conceito de proporcionalidade através de resolução de problemas de Geometria}

Nesta seção descrevemos e analisamos os dados coletados em um dos momentos de reflexão e discussão sobre a Metodologia de Ensino-Aprendizagem-Avaliação através de resolução de problemas. Nesse encontro o objetivo era verificar como (futuros) professores em formação inicial aplicam o conceito de proporcionalidade (propriedade fundamental das proporções) para calcular o valor de termos desconhecidos em problemas que envolvem o Teorema de Tales e, com isso, construir novos conhecimentos matemáticos. 
Um dos problemas que utilizamos foi o seguinte:

A planta abaixo mostra as medidas de três lotes que têm frente para a rua A e para a rua $\mathrm{B}$. As divisas laterais são perpendiculares à rua $\mathrm{A}$. Quais são as medidas de $\mathrm{x}$ e y indicadas na figura?

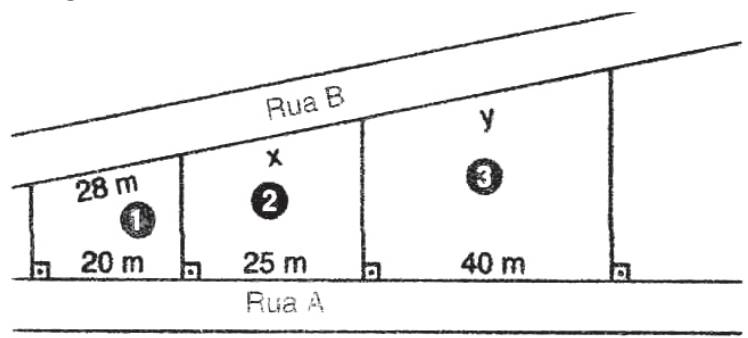

Fonte: Andrini; Vasconcellos (2006)

Para que os (futuros) professores, participantes da pesquisa, pudessem ter uma melhor compreensão da metodologia, sugerimos que utilizassem as etapas sugeridas por Allevato e Onuchic (2009). Primeiramente preparamos o problema de acordo com o conteúdo a ser explorado (etapa 1), ou seja, a proporcionalidade e o Teorema de Tales. Ao entregar o problema aos alunos, pedimos que fizessem uma leitura individual (etapa 2), tentando compreender o enunciado, detectar dúvidas e se preparar para a resolução. Então fizemos uma leitura coletiva (etapa 3), para esclarecer eventuais dúvidas. Então cada participante tentou resolver o problema individualmente. Apesar de ser sugerido pelas autoras que essa etapa fosse realizada em grupo (etapa 4), optamos por esse método para que pudéssemos observar melhor (etapa 5) as atitudes de cada aluno. Após terem solucionado o problema, cada aluno fez o registro na lousa das resoluções e soluções encontradas (etapa 6) e, em seguida, realizamos uma plenária (etapa 7), buscando um consenso em relação às soluções (etapa 8). Finalmente, o pesquisador fez a formalização do conteúdo abordado no problema (etapa 9), registrando na lousa uma apresentação organizada e estruturada em linguagem matemática do conceito de proporcionalidade, e abordou o método que os participantes utilizaram para solucionar o problema: a regra de três.

Observamos, após a leitura individual e coletiva, que o enunciado ficou claro e que os (futuros) professores tiveram facilidade em resolver o problema. Durante as discussões na plenária, notamos que os participantes utilizaram a regra de três para solucionar o problema. Dois deles comentaram que, além da regra de três, utilizaram o conceito de proporcionalidade (como igualdade entre duas razões) para solucionar o problema. 
Nenhum dos participantes fez menção ao Teorema de Tales, embora ele possua diversas aplicações no cotidiano e em outras áreas, que evidenciam sua importância. Através dele podemos perceber que retas paralelas cortadas por transversais formam segmentos correspondentes proporcionais (DANTE, 2004). Portanto, podemos compreender o Teorema através do problema proposto, e até demonstrá-lo generalizando esse resultado. Com base na planta apresentada no problema, solicitamos aos alunos que calculassem as medidas dos lados $\mathrm{x}$ e $\mathrm{y}$. Podemos perceber que os segmentos paralelos que separam os lotes 1, 2 e 3 são cortados pelas ruas transversais A e B. Portanto, a planta "satisfaz" a relação de Tales e, sendo assim, os alunos poderiam utilizá-lo para solucionar o problema.

Embora alguns participantes tivessem conseguido perceber a proporcionalidade existente no problema, não relacionaram esse fato ao chamado Teorema de Tales, ou seja, eles conhecem o resultado, mas não o conhecem com o nome de Teorema de Tales. Alguns, ainda, não tinham clareza sobre o próprio conceito de proporcionalidade, conforme podemos observar a seguir, nas resoluções apresentadas ${ }^{3}$ :

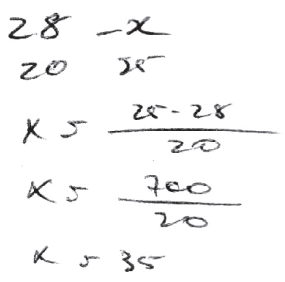

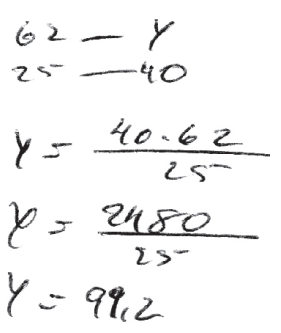

Resolução apresentada por ADR3

O aluno ADR3 comentou que, de fato, esse foi um problema para ele, pois encontrou dificuldades para encontrar a solução. Percebemos na resolução apresentada pelo aluno que ele não utilizou a definição de proporcionalidade, ou seja, ele não registrou a igualdade das duas razões, conforme havia afirmado em conversa com o pesquisador para esclarecimento de sua resolução, indo direto para a regra de três. No entanto, a solução encontrada para o termo desconhecido (y) não foi correta, conforme podemos observar na resolução acima apresenta por esse aluno.

3. Para mantermos a identidade desses (futuros) professores, usaremos pseudônimos: ADR1, ADR2, ADR3..., para identificá-los. 
Mais uma resolução apresenta foi:
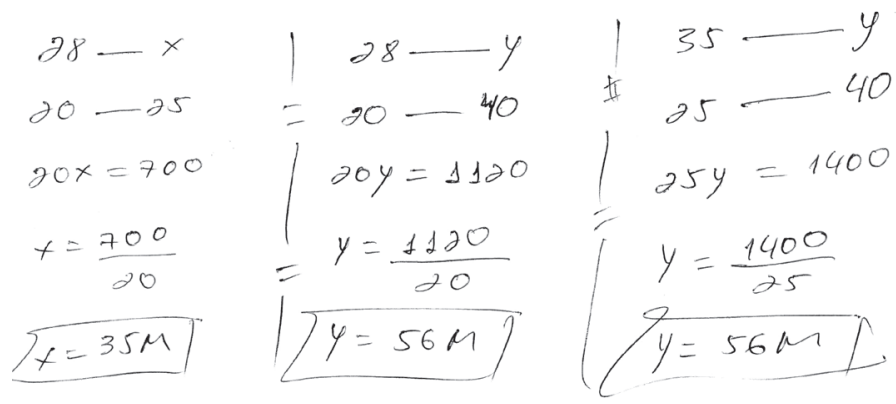

\section{Resolução apresentada por AR5}

Em conversa com o aluno ADR5 após a resolução do problema, ele afirmou que o mesmo foi de fácil interpretação, e que utilizou o conceito de proporcionalidade e a regra de três para identificar e resolver o problema. Analisando a resolução apresentada (acima) por este aluno, percebemos que ele também não registrou o conceito de proporcionalidade. Certamente o conceito como igualdade entre duas razões não está presente em suas mentes e eles não o escrevem; esse aluno também foi direto para regra de três para encontrar os valores desconhecidos. Portanto, não utilizou o conceito de proporcionalidade, conforme havia afirmado. Percebemos, também, que o aluno procurou confirmar a solução encontrada para " $y$ " de duas maneiras. Isto nos leva a crer que ele não tinha certeza e buscou uma confirmação quanto ao valor encontrado de imediato.

A seguir apresentamos a resolução dos alunos ADR1 e ADR4, em que podemos observar que partiram do conceito de proporcionalidade, escrevendo a igualdade entre as duas razões, utilizando, em seguida, a regra de três para solucionar o problema. 


$$
\begin{aligned}
& z \rightarrow(1) \Rightarrow z=28 \mathrm{~m} \\
& x \rightarrow \text { (2) } \Rightarrow x=35 \mathrm{~m} \quad \frac{x}{25}=\frac{y}{40} \\
& \begin{array}{l}
y x(3) \Rightarrow y=56 n \\
z+x+y=0
\end{array} \\
& \frac{z}{20}=\frac{x}{25} \\
& 25 y=1400 \\
& y=\frac{1400}{25} \\
& \frac{28}{20}=\frac{x}{25} \\
& 20 x=700 \\
& \alpha=\frac{20 \phi}{2 \phi} \\
& x=35 \mu
\end{aligned}
$$

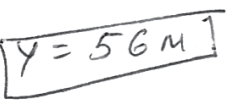

Resolução apresentada por ADR1

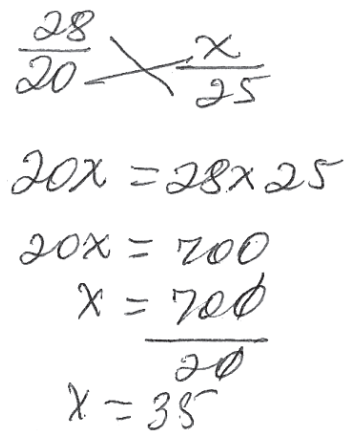

$$
\begin{aligned}
& \frac{35}{25} \frac{y}{40} \\
& 25 y=1400 \\
& y=\frac{1400}{25} \\
& y=64
\end{aligned}
$$

\section{Resolução apresentada por ADR4}

Segundo os (futuros) professores ADR1 e ADR4, entender o problema foi fácil, e para solucioná-lo registraram o conceito de proporcionalidade e utilizaram a regra de três. Além disso, ADR4 acrescentou que para ele não foi um problema, pois foi muito fácil encontrar a solução. No entanto, deve-se destacar que ele não encontrou a solução correta para o valor do termo desconhecido denominado de "y", devido a um erro de cálculo. 
Vale ressaltar que em sua resolução ADR4 fez uma indicação de produto cruzado, que é um procedimento da regra de três. No entanto, observamos que ele não utilizou o sinal de igualdade nem na primeira, nem na segunda coluna. Isso nos leva a crer que o mesmo não pensou na igualdade entre duas razões, ou seja, não fez associação ao conceito de proporcionalidade, apesar de ter comentando que fez esse registro.

Observamos, ainda, nas soluções apresentadas pelos alunos, que eles têm a regra de três como procedimento padrão para resolução de problemas de proporcionalidade. Isto acontece, provavelmente, porque no ensino básico o conceito de proporcionalidade é apresentado aos alunos realmente priorizando a regra de três como meio para solucionar um problema.

Em particular, com relação ao estudo de proporcionalidade, Curcio e Bezuk (1994) afirmam que "as soluções de muitos problemas envolvendo medidas, geometria, álgebra, probabilidade e estatística requerem o conhecimento de, e a facilidade com, números racionais e proporções" (CURCIO; BEZUK, 1994, p. 2). Porém, o que percebemos nas situações escolares é que não raro os alunos conduzem suas estratégias de resolução baseadas principalmente nos dados numéricos que o problema apresenta, não levando, muitas vezes, em consideração os dados relacionais necessários para a compreensão da situação e resolução correta.

Ao final da atividade, durante a plenária, esses aspectos foram abordados: (re) construímos o conceito de proporcionalidade, discutindo quando duas grandezas são de fato proporcionais; as estratégias ou formas de resolução (regra de três, divisões sucessivas, montagem de tabelas) e a relação existente entre a proporcionalidade e o Teorema de Tales.

\section{Reflexões Finais}

No início deste trabalho, trazíamos uma inquietação muito grande com relação ao ensino de proporcionalidade e à formação dos (futuros) professores para ensinar esse tema a seus alunos através de resolução de problemas de Geometria.

Com relação ao nosso objetivo inicial, nossa pesquisa revelou que os (futuros) professores tinham poucos conhecimentos com relação ao conteúdo estudado, principalmente em fazer conexão com a Geometria (Teorema de Tales). A falta de conhecimentos não era apenas com relação aos conteúdos; eles também tinham dúvidas em relação a "quando" (ano escolar) e "como" deveriam ensinar.

Com relação a isso, retomamos as idéias de Tinoco (1996) com relação à necessidade de um trabalho em que a Matemática seja abordada com uma visão mais unificada, estabelecendo relações entre os diversos conteúdos e, em particular, entre a Geometria e as demais áreas. 
Além, disso, na constatação da construção dos conhecimentos que os (futuros) professores construíram, evidenciou-se o potencial da resolução de problemas como metodologia de ensino, ou seja, como um meio para aprender Matemática e não apenas para aplicá-la. Tendo constituído-se, de fato, em problemas, as atividades propostas envolveram os participantes na busca pela solução, na exploração das possibilidades de resolução, e na (re)construção de conhecimentos sobre proporcionalidade e Geometria.

No início das discussões os (futuros) professores tiveram resistência com relação à Metodologia de Ensino-Aprendizagem-Avaliação de Matemática através da Resolução de Problemas. Inicialmente, eles tiveram uma impressão equivocada da metodologia, dizendo que, essa metodologia era boa, mas para o professor, que não precisa "explicar nada para os alunos". Mas, ao vivenciarem na prática, perceberam que o professor trabalha muito: precisa preparar um problema que atinja os objetivos propostos para o conteúdo a ser estudado, criar e manter um ambiente estimulante para as aulas, esclarecer dúvidas e incentivar os alunos a resolvê-lo e, no final, ainda, formalizar o conteúdo. Essa é uma das diferenças com relação ao ensino tradicional em que o professor inicia sua aula formalizando o conteúdo.

As mudanças foram acontecendo de forma lenta e gradual durante os encontros. E logo eles comentaram que utilizar essa metodologia é uma maneira nova de se trabalhar com a Matemática, mas que, de imediato, para quem está tendo o primeiro contato, parece ser mais difícil.

Ainda acrescentaram que essa resistência ocorre por não estarem acostumados a ver professores ensinando dessa maneira. No entanto, eles consideram que essa metodologia deve ser implantada a longo prazo, mas acreditam que essa seria mesmo uma boa maneira de o aluno aprender e se interessar pela Matemática. O aluno será estimulado a raciocinar e a ir em busca de novas descobertas

Os (futuros) professores, no início das discussões, tinham pouca segurança para expor suas ideias na plenária, mas aos poucos foram percebendo a importância dessa discussão antes da formalização do conteúdo. Com o passar do tempo, foram ficando mais seguros com relação a metodologia e comentaram que pretendem desenvolvê-la com seus (futuros) alunos.

Ressaltamos que, apesar de não terem sido o objetivo de nosso trabalho, a mudança do ponto de vista dos (futuros) professores em relação à metodologia $\mathrm{e}$ a resolução de problemas, que aparece com grande ênfase nos dados, emergiu no decorrer das análises, ou seja, após a realização do trabalho, constituindo-se em um desdobramento que consideramos bastante relevante. Elas sinalizam para a possibilidade de mudanças nas práticas desses (futuros) professores e para a incorporação de novas metodologias ao seu trabalho com Matemática em sala de aula. 
Foi possível perceber que o trabalho compartilhado possibilitou a mobilização e a produção de novos saberes, tanto relativos aos conhecimentos de conteúdos que foram discutidos, quanto os relativos aos conhecimentos metodológicos desses conteúdos.

Foram elementos essenciais tanto a compreensão da Metodologia de Ensino-Aprendizagem-Avaliação de Proporcionalidade através da Resolução de Problemas, como a ampliação dos conhecimentos sobre proporcionalidade. No entanto, é necessário avançar, tentar, redescobrir, ler, produzir e sobretudo acreditar.

\section{Referências}

ALLEVATO. N. S. G.; ONUCHIC; L. R. Ensinando Matemática na Sala de Aula através de resolução de Problemas. Boletim Gepem, n. 55, p. 1-19. 2009. Disponível em http://www.ufrrj.br/SEER/index.php/gepem/article/view/54/87. Acesso em 11. mai. 2011.

ANDRINI, A; VASCONCELlOS, M. J. Novo Praticando Matemática, v.4, 1 ed. São Paulo: Editora do Brasil, 2006.

BRASIL - Ministério da Educação e do Desporto. Parâmetros Curriculares Nacionais - Matemática $1^{\circ}$ e $2^{\circ}$ ciclos: Matemática. Brasília, 1998.

COSTA, M. S.; ALLEVATO, N. S. G. Resolução de Problemas: Fixação de Conteúdos Matemáticos ou Metodologia de Ensino? Anais do V CIEM - Congresso Internacional de Ensino da Matemática, Canoas: Universidade Luterana do Brasil, 2010.

COSTA, M. S.; ALLEVATO, N. S. G. O Conceito de Proporcionalidade na Resolução de Problemas de Geometria. Anais do XIII EBRAPEM - Encontro Brasileiro de Estudantes de Pós-Graduação em Educação Matemática, Goiânia: Universidade Federal de Goiânia, 2009.

CURCIO. F. R.; BEZUK. N. S. Understandig rational numbers and proportions. In: Curriculum and evaluation standards for school mathematics addenda series. Grades 5-8. Reston: NCTM, 1994.

DANTE, L. R. Tudo é matemática. $7^{\mathrm{a}}$ série. São Paulo: Ática, 2004.

GOLDENBERG, M. A arte de pesquisar: como fazer pesquisa qualitativa em ciências sociais. Rio de Janeiro: Record, 2007. 
LÜDKE, M.; ANDRÉ, M. E. D. A. Pesquisa em educação: abordagens qualitativas. São Paulo: EPU, 1986.

MARANHÃO (Estado). Secretaria de Estado da Educação. Secretaria Adjunta de Ensino. Referenciais Curriculares: Ensino Médio: Matemática. São Luís, 2007.

NCTM - National Council of Teachers of Mathematics. An Agenda for Action. Reston: NCTM, 1980.

NUNES. C. B. O Processo Ensino-Aprendizagem-Avaliação de Geometria através da Resolução de Problemas: perspectivas didático-matemáticas na formação inicial de professores de matemática. Tese (Doutorado em Educação Matemática). Instituto de Geociências e Ciências Exatas, UNESP - Rio Claro, 2010.

OLIVEIRA, I. A. F. G.; SANTOS. M. C. Problemas de proporção: uma análise da apropriação do seu significado. Anais do IV EPEM - Encontro Pernambucano de Educação Matemática. Recife: Universidade Federal de Pernambuco, 1999.

ONUCHIC, L. R.; ALLEVATO, N. S. G. Formação de Professores - Mudanças Urgentes na Licenciatura em Matemática. In: FROTA, M. C. R.; NASSER, 1. (orgs). Educação Matemática no Ensino Superior: pesquisas e debates. Recife: SBEM, 2009. p. 169-187.

ONUCHIC, L. R. Ensino-Aprendizagem de Matemática através de resolução de problemas. In: BICUDO, M. A. V. Pesquisas em Educação Matemática. São Paulo: Editora UNESP, 1999, p. 199-220.

TINOCO, L. A. A. Razões e Proporções. Rio de Janeiro: UFRJ, 1996.

VAN DE WALLE, J. A. Matemática no ensino fundamental: formação de professores e aplicação em sala de aula. Tradução: Paulo Henrique Colonese. 6. ed. Porto Alegre: Artmed, 2009.

VIANNA, C. R. Resolução de Problemas. In: Futuro Congressos e Eventos. (Org.). Temas em Educação I - Livro das Jornadas. Curitiba: Futuro Congressos e Eventos, 2002, p. 401-410. 